Wat er-sol ubl e mel at oni ns: Synt heses of mel at oni ns carrying a gl ycosyl group at the 1- positi on

\begin{tabular}{|l|l|}
\hline 著者 & $\begin{array}{l}\text { I waki Takako, Fuj i t a Yasuaki , Yanada Fumi o, } \\
\text { Sonei Nasanor i }\end{array}$ \\
\hline $\begin{array}{l}\text { j our nal or } \\
\text { publ i cat i on t i t l e }\end{array}$ & Het er ocycl es \\
\hline vol une & 60 \\
\hline number & 6 \\
\hline page r ange & $1411-1418$ \\
\hline year & 2003- 06-01 \\
\hline URL & ht t p: //hdl . handl e. net /2297/4376 \\
\hline
\end{tabular}


Received, 14th February, 2003, Accepted, 4th April, 2003, Published online, 7th April, 2003

\title{
WATER-SOLUBLE MELATONINS: SYNTHESES OF MELATONINS CARRYING A GLYCOSYL GROUP AT THE 1-POSITION ${ }^{1}$
}

\section{Takako Iwaki, Yasuaki Fujita, Fumio Yamada, and Masanori Somei*}

\author{
Faculty of Pharmaceutical Sciences, Kanazawa University, \\ 13-1 Takara-machi, Kanazawa 920-0934, Japan \\ e-mail address: somei@mail.p.kanazawa-u.ac.jp
}

\begin{abstract}
D-Xylopyranosyl)- (2a), 1-( $\beta$-D-glucopyranosyl)- (2b), 1-( $\beta$-Dgalactopyranosyl)- (2c), and 1-( $\alpha$-D-arabinopyranosyl)melatonins (3b) are prepared as water-soluble melatonins starting from melatonin.
\end{abstract}

Regulation of the circadian rhythms, ${ }^{2}$ inhibition of Alzheimer $\beta$-fibrillogenesis, ${ }^{3 a}$ anti-aging properties relating to radical scavenging, ${ }^{3 b}$ antiproliferative effect on melanoma cells, ${ }^{3 \mathrm{c}}$ and so on $^{3}$ are well known biological activities ${ }^{3}$ reported for melatonin ${ }^{2}(1$, Scheme 1), a pineal gland hormone.

In our continuing project ${ }^{4}$ for developing new biologically active compounds based on indole nucleus, we have thus far succeeded in finding our own synthetic method ${ }^{4}$ for melatonin (1). With $\mathbf{1}$ in hand, we have now reached to the stage ${ }^{1 c}$ for creating water-soluble melatonin ${ }^{1 c}$ in order to examine whether such change in physical properties of $\mathbf{1}$ has a substantial effect on its biological activities. In this paper, we wish to report the results of our trial to produce 1-glycosylmelatonin derivatives $(\mathbf{2} \mathbf{a}-\mathbf{c}, \mathbf{3 b})$, chosen as our targets among various candidates for water-soluble melatonins.

Preobrazhenskaya ${ }^{5}$ and co-workers reported a synthetic method for 1-glycosylindoles without using any protecting group, consisting of heating indolines with an appropriate sugar component, followed by DDQ oxidation. So, we applied the procedure to meet our ends.

Melatonin $^{4}(\mathbf{1})$, prepared through biologically active 1-hydroxytryptamines, ${ }^{6}$ was first converted to $2,3-$ dihydromelatonin (4) as reported ${ }^{4 \mathrm{~d}, \mathrm{e}}$ previously by reduction with $\mathrm{Et}_{3} \mathrm{SiH}^{7}$ in $\mathrm{CF}_{3} \mathrm{COOH}$ in $99 \%$ yield. ${ }^{4 \mathrm{e}}$ Although treatment of 4 with 3-mol eq. of D-xylose in refluxing $\mathrm{MeOH}$ afforded 1-glycosyl-2,3dihydromelatonin, its isolation in pure state was difficult. The reaction mixture was therefore acetylated with $\mathrm{Ac}_{2} \mathrm{O}$-pyridine to afford a 1:1 mixture of diastereomers (5a) in $85 \%$ yield. Oxidation of the mixture with 1.2-mol eq. of DDQ in dioxane at room temperature produced $6 \mathbf{a}$ as a single isomer in $56 \%$ yield. 
To the contrary to the expected improvement, employment of 2-mol eq. of DDQ dropped the yield of $\mathbf{6 a}$ down to $46 \%$ with formation of tar.

\section{Scheme 1}

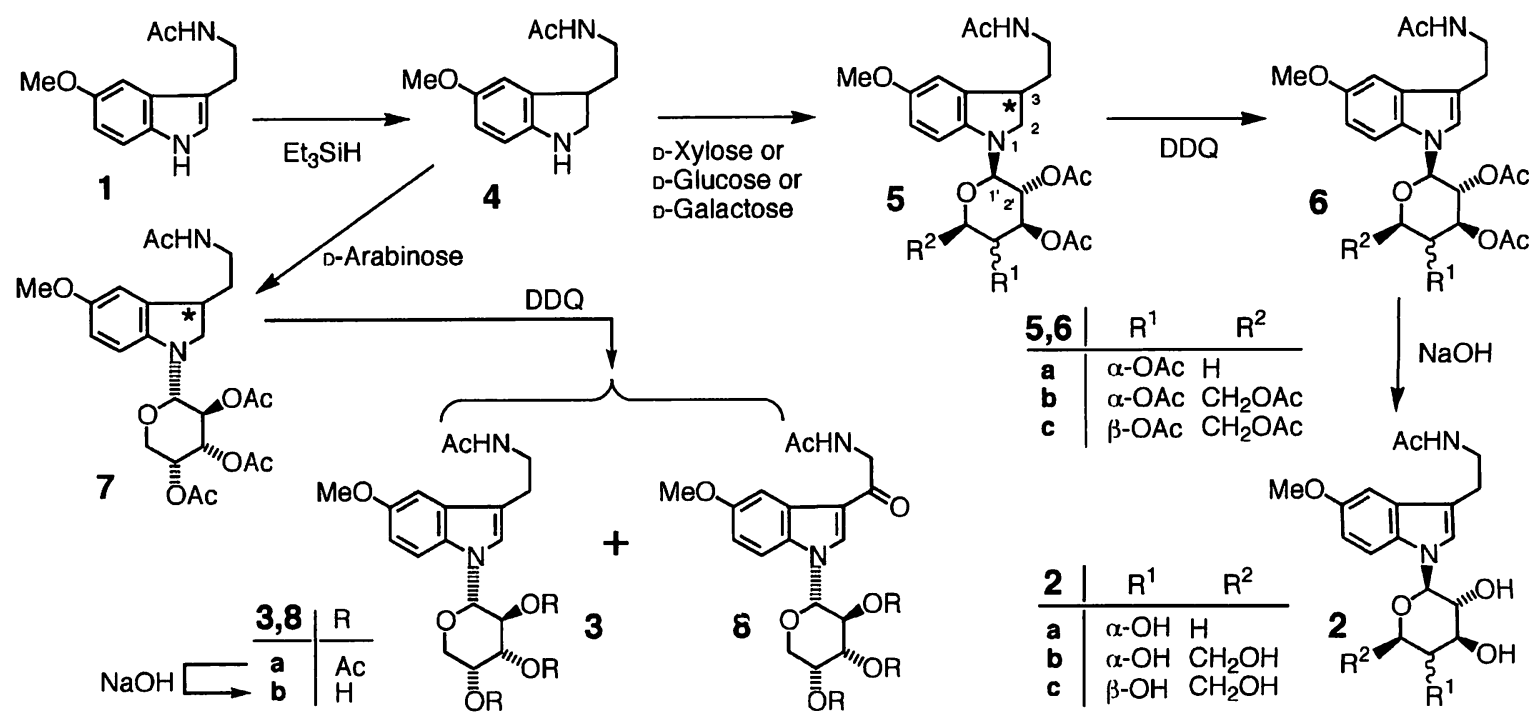

Similar reactions of 4 with D-glucose and D-galactose, followed by treatment with $\mathrm{Ac}_{2} \mathrm{O}$-pyridine, afforded $\mathbf{5 b}$ and $\mathbf{5 c}$ in 97 and $85 \%$ yields, respectively. Oxidations of $\mathbf{5 b}$ and $\mathbf{5 c}$ with $1-\mathrm{mol}$ eq. of DDQ produced $6 \mathbf{b}$ and $6 \mathbf{c}$ in the respective yields of 87 and $80 \%$. In these cases, the employment of 2-mol eq. of DDQ decreased the yields of $6 \mathbf{b}$ and $\mathbf{6 c}$ to 55 and $45 \%$ yields, respectively. The anomeric (C-1') protons in the ${ }^{1} \mathrm{H}-\mathrm{NMR}$ spectra of $\mathbf{6 a}-\mathbf{c}$ clearly appeared as a doublet with a coupling constant of $9 \mathrm{~Hz}$. Based on these data, their structures are confirmed to be 1-( $\beta$-D-glycosyl)melatonin and the diastereomers $(\mathbf{5 a}-\mathbf{c})$ arise from the stereochemistry at the indole 3-position.

In the case of $\mathrm{D}$-arabinose, 7 was obtained in $95 \%$ yield by the reaction with 4 , followed by treatment with $\mathrm{Ac}_{2} \mathrm{O}$-pyridine. An interesting fact is the formation of $\mathbf{8 a}$ in $10 \%$ yield together with $43 \%$ yield of 3a when the oxidation of 7 was carried out with 1.2-mol eq. of DDQ. 2-mol eq. of DDQ raised the yield of $8 \mathbf{a}$ up to $34 \%$ together with $4 \%$ yield of $\mathbf{3 a}$. The anomeric protons of $8 \mathbf{a}$ and $\mathbf{3 a}$ appear as a doublet with a coupling constant of $9 \mathrm{~Hz}$. Therefore, their trans-diaxial relationship to the neighboring C-2' proton is proved. Since D-arabinose has $\beta$-hydroxy group at the C-2' position, structures of 8a and 3a are confirmed to be 1-( $\alpha$-D-glycosyl)melatonin derivatives. The difference in the anomeric configuration between 7 and $\mathbf{5 a}-\mathbf{c}$ seems to be the cause of their differences in the oxidation behavior.

Hydrolysis of ester group in $6 \mathbf{a}$ was carried out with $8 \%$ aqueous $\mathrm{NaOH}$ to give the desired 1-( $\beta$-Dxylopyranosyl)melatonin (2a) in $83 \%$ yield. Similarly, alkaline hydrolysis of $\mathbf{6 b}, \mathbf{6 c}, 3 \mathbf{a}$, and $8 \mathbf{a}$ provided $\mathbf{2 b}, \mathbf{2 c}, \mathbf{3 b}$, and $\mathbf{8 b}$ in $90,93,87$, and $81 \%$ yields, respectively. 
Biological evaluations of 1-glycosylmelatonins and syntheses of other types of water-soluble melatonins are now in progress.

\section{EXPERIMENTAL}

IR spectra were determined with a Shimadzu IR-420 or HORIBA FT-720 spectrophotometer and ${ }^{1} \mathrm{H}-$ NMR spectra with a JEOL GSX-500 spectrometer with tetramethylsilane as an internal standard. MS spectra were recorded on a JEOL SX-102A spectrometer. PTLC was performed on Merck Kiesel-gel $\mathrm{GF}_{254}$ (Type 60)( $\left.\mathrm{SiO}_{2}\right)$. Column chromatography was performed on silica gel $\left(\mathrm{SiO}_{2}, 100-200\right.$ mesh, from Kanto Chemical Co. Inc.) throughout the present study.

1-( $\beta$-D-Triacetylxylopyranosyl)-2,3-dihydromelatonin (5a) from 4 - D-Xylose (112.5 mg, 0.75 mmol) was added to a solution of $4(58.5 \mathrm{mg}, 0.25 \mathrm{mmol})$ in $\mathrm{MeOH}(4.0 \mathrm{~mL})$ and the mixture was refluxed for $2 \mathrm{~h}$ with stirring. The solvent was evaporated under reduced pressure to leave an oil, which was dissolved in pyridine $(3.0 \mathrm{~mL}, 37.0 \mathrm{mmol})$. To the resultant solution, $\mathrm{Ac}_{2} \mathrm{O}(1.5 \mathrm{~mL}, 15.9 \mathrm{mmol})$ was added and the mixture was stirred at $\mathrm{rt}$ for $2.5 \mathrm{~h}$. The solvent was evaporated under reduced pressure. After addition of $\mathrm{H}_{2} \mathrm{O}$ to the residue, the whole was extracted with $\mathrm{CHCl}_{3}-\mathrm{MeOH}(95: 5$, v/v). The extract was washed with $10 \% \mathrm{NH}_{4} \mathrm{Cl}$ and brine, dried over $\mathrm{Na}_{2} \mathrm{SO}_{4}$ and evaporated under reduced pressure to leave an oil, which was column-chromatographed on $\mathrm{SiO}_{2}$ successively with $\mathrm{CHCl}_{3}-\mathrm{MeOH}(99: 1$, v/v) and $\mathrm{CHCl}_{3}-\mathrm{MeOH}(95: 5, \mathrm{v} / \mathrm{v})$ to give $\mathbf{5 a}\left(105.3 \mathrm{mg}\right.$, 85\%). 5a: pale yellow oil. IR (film): $1754,1654 \mathrm{~cm}^{-1}$. ${ }^{1} \mathrm{H}-\mathrm{NMR}\left(\mathrm{CDCl}_{3}\right)$ 8: 1.59-1.76 (2H, m), $1.93(3 / 2 \mathrm{H}, \mathrm{s}), 1.96(3 / 2 \mathrm{H}, \mathrm{s}), 1.99(3 / 2 \mathrm{H}, \mathrm{s}), 2.01(3 / 2 \mathrm{H}, \mathrm{s})$, $2.04(3 \mathrm{H}, \mathrm{s}), 2.05(3 / 2 \mathrm{H}, \mathrm{s}), 2.06(3 / 2 \mathrm{H}, \mathrm{s}), 3.15-3.40(5 \mathrm{H}, \mathrm{m}), 3.66(1 / 2 \mathrm{H}, \mathrm{t}, J=7.8 \mathrm{~Hz}), 3.71(1 / 2 \mathrm{H}, \mathrm{t}$, $J=7.5 \mathrm{~Hz}), 3.73(3 \mathrm{H}, \mathrm{s}), 4.04-4.09(1 \mathrm{H}, \mathrm{m}), 4.81(1 / 2 \mathrm{H}, \mathrm{d}, J=9.0 \mathrm{~Hz}), 4.83(1 / 2 \mathrm{H}, \mathrm{d}, J=9.0 \mathrm{~Hz})$, 4.96-5.02 (1H, m), $5.17(1 / 2 \mathrm{H}, \mathrm{t}, J=9.0 \mathrm{~Hz}), 5.19(1 / 2 \mathrm{H}, \mathrm{t}, J=9.0 \mathrm{~Hz}), 5.32(1 / 2 \mathrm{H}, \mathrm{t}, J=9.0 \mathrm{~Hz}), 5.33$ $(1 / 2 \mathrm{H}, \mathrm{t}, J=9.0 \mathrm{~Hz}), 5.57(1 / 2 \mathrm{H}$, br t,$J=5.2 \mathrm{~Hz}), 5.67(1 / 2 \mathrm{H}$, br t,$J=5.2 \mathrm{~Hz}), 6.50(1 / 2 \mathrm{H}, \mathrm{d}, J=8.6 \mathrm{~Hz})$, $6.51(1 / 2 \mathrm{H}, \mathrm{d}, J=8.6 \mathrm{~Hz}), 6.66(1 / 2 \mathrm{H}, \mathrm{dd}, J=8.6,2.5 \mathrm{~Hz}), 6.67(1 / 2 \mathrm{H}, \mathrm{dd}, J=8.6,2.5 \mathrm{~Hz}), 6.70(1 / 2 \mathrm{H}, \mathrm{d}$, $J=2.5 \mathrm{~Hz}), 6.75(1 / 2 \mathrm{H}, \mathrm{d}, J=2.5 \mathrm{~Hz})$. HRMS $m / z$ : Calcd for $\mathrm{C}_{24} \mathrm{H}_{32} \mathrm{~N}_{2} \mathrm{O}_{9}: 492.2108$. Found: 492.2104. $[\alpha]_{\mathrm{D}}^{25}+8.15^{\circ}(\mathrm{c}=0.135, \mathrm{MeOH})$.

1-( $\beta$-D-Tetraacetylglucopyranosyl)-2,3-dihydromelatonin (5b) from 4 - In the same procedure as described in the preparation of $\mathbf{5 a}$, D-glucose (76.9 mg, $0.43 \mathrm{mmol}), 4$ (20.1 $\mathrm{mg}, 0.086 \mathrm{mmol}), \mathrm{MeOH}$ $(1.5 \mathrm{~mL})$, pyridine $(1.5 \mathrm{~mL}, 18.5 \mathrm{mmol})$ and $\mathrm{Ac}_{2} \mathrm{O}(0.75 \mathrm{~mL}, 7.9 \mathrm{mmol})$ were used. Columnchromatography was performed on $\mathrm{SiO}_{2}$ with $\mathrm{CHCl}_{3}$ to give $\mathbf{5 b}(46.9 \mathrm{mg}, 97 \%)$. $5 \mathbf{b}$ : colorless oil. IR (film): $1751,1655 \mathrm{~cm}^{-1} .{ }^{1} \mathrm{H}-\mathrm{NMR}\left(\mathrm{CDCl}_{3}\right) \delta: 1.61-1.67(1 \mathrm{H}, \mathrm{m}), 1.69-1.79(1 \mathrm{H}, \mathrm{m}), 1.94(3 / 2 \mathrm{H}, \mathrm{s})$, $1.95(3 / 2 \mathrm{H}, \mathrm{s}), 1.99(3 \mathrm{H}, \mathrm{s}), 2.00(3 / 2 \mathrm{H}, \mathrm{s}), 2.01(3 / 2 \mathrm{H}, \mathrm{s}), 2.03-2.04(3 / 2 \mathrm{Hx} 4$, each s), 3.15-3.40 (4H, $\mathrm{m}), 3.68(1 \mathrm{H}, \mathrm{t}, J=8.8 \mathrm{~Hz}), 3.74(3 \mathrm{H}, \mathrm{s}), 3.71-3.76(1 \mathrm{H}, \mathrm{m}), 4.03(1 / 2 \mathrm{H}, \mathrm{dd}, J=12.2,2.4 \mathrm{~Hz}), 4.06(1 / 2 \mathrm{H}$, 
dd, $J=12.2,2.4 \mathrm{~Hz}), 4.24(1 / 2 \mathrm{H}$, dd, $J=12.2,3.2 \mathrm{~Hz}), 4.26(1 / 2 \mathrm{H}$, dd, $J=12.2,3.2 \mathrm{~Hz}), 4.88(1 / 2 \mathrm{H}, \mathrm{d}$, $J=9.1 \mathrm{~Hz}), 4.89(1 / 2 \mathrm{H}, \mathrm{d}, J=9.1 \mathrm{~Hz}), 5.07(1 / 2 \mathrm{H}, \mathrm{t}, J=9.0 \mathrm{~Hz}), 5.08(1 / 2 \mathrm{H}, \mathrm{t}, J=9.0 \mathrm{~Hz}), 5.23(1 / 2 \mathrm{H}, \mathrm{t}$, $J=9.0 \mathrm{~Hz}), 5.24(1 / 2 \mathrm{H}, \mathrm{t}, J=9.0 \mathrm{~Hz}), 5.33(1 / 2 \mathrm{H}, \mathrm{t}, J=9.0 \mathrm{~Hz}), 5.34(1 / 2 \mathrm{H}, \mathrm{t}, J=9.0 \mathrm{~Hz}), 5.56(1 / 2 \mathrm{H}$, br t, $J=5.0 \mathrm{~Hz}), 5.69(1 / 2 \mathrm{H}$, br t, $J=5.0 \mathrm{~Hz}), 6.48(1 / 2 \mathrm{H}, \mathrm{d}, J=8.5 \mathrm{~Hz}), 6.50(1 / 2 \mathrm{H}, \mathrm{d}, J=8.5 \mathrm{~Hz}), 6.66(1 \mathrm{H}, \mathrm{dd}$, $J=8.5,2.4 \mathrm{~Hz}), 6.71(1 / 2 \mathrm{H}, \mathrm{d}, J=2.4 \mathrm{~Hz}), 6.75(1 / 2 \mathrm{H}, \mathrm{d}, J=2.4 \mathrm{~Hz})$. HRMS $m / z$ : Calcd for $\mathrm{C}_{27} \mathrm{H}_{36} \mathrm{~N}_{2} \mathrm{O}_{11}$ : 564.2319. Found: $564.2324 .[\alpha]_{D}{ }^{24}-5.76^{\circ}(\mathrm{c}=0.10, \mathrm{MeOH})$.

1-( $\beta$-D-Tetraacetylgalactopyranosyl)-2,3-dihydromelatonin (5c) from 4 - In the same procedure as described in the preparation of 5a, D-galactose (231.2 mg, $1.28 \mathrm{mmol}), 4(100.2 \mathrm{mg}, 0.43 \mathrm{mmol}), \mathrm{MeOH}$ $(6.0 \mathrm{~mL})$, pyridine $(8.0 \mathrm{~mL}, 99.0 \mathrm{mmol})$, and $\mathrm{Ac}_{2} \mathrm{O}(4.0 \mathrm{~mL}, 42.3 \mathrm{mmol})$ were used. Columnchromatography was performed on $\mathrm{SiO}_{2}$ successively with $\mathrm{CHCl}_{3}$ and $\mathrm{CHCl}_{3}-\mathrm{MeOH}(99: 1 \mathrm{v} / \mathrm{v})$ to give 5c $(205.4 \mathrm{mg}, 85 \%)$. 5c: colorless oil. IR (film): $1745,1653 \mathrm{~cm}^{-1} .{ }^{1} \mathrm{H}-\mathrm{NMR}\left(\mathrm{CDCl}_{3}\right) \delta: 1.61-1.69(1 \mathrm{H}$, m), $1.70-1.80(1 \mathrm{H}, \mathrm{m}), 1.94(3 / 2 \mathrm{H}, \mathrm{s}), 1.96(3 / 2 \mathrm{H}, \mathrm{s}), 1.97(3 / 2 \mathrm{H}, \mathrm{s}), 1.98(3 / 2 \mathrm{H}, \mathrm{s}), 1.99(3 / 2 \mathrm{H}, \mathrm{s})$, $2.01-2.02$ (3/2Hx3, each s), $2.17(3 / 2 \mathrm{H}, \mathrm{s}), 2.19(3 / 2 \mathrm{H}, \mathrm{s}), 3.16-3.44(4 \mathrm{H}, \mathrm{m}), 3.71-3.77(1 \mathrm{H}, \mathrm{m})$, $3.75(3 \mathrm{H}, \mathrm{s}), 3.96(1 \mathrm{H}, \mathrm{t}, J=6.7 \mathrm{~Hz}), 4.02-4.13(2 \mathrm{H}, \mathrm{m}), 4.83(1 / 2 \mathrm{H}, \mathrm{d}, J=9.0 \mathrm{~Hz}), 4.85(1 / 2 \mathrm{H}, \mathrm{d}, J=9.0$ $\mathrm{Hz}), 5.14(1 / 2 \mathrm{H}, \mathrm{dd}, J=9.0,3.4 \mathrm{~Hz}), 5.16(1 / 2 \mathrm{H}, \mathrm{dd}, J=9.0,3.4 \mathrm{~Hz}), 5.39-5.40(1 / 2 \mathrm{Hx} 2$, each t, $J=9.0$ $\mathrm{Hz}), 5.43(1 \mathrm{H}, \mathrm{m}), 5.54(1 / 2 \mathrm{H}, \mathrm{br} \mathrm{t}), 5.61(1 / 2 \mathrm{H}, \mathrm{br} \mathrm{t}), 6.50(1 \mathrm{H}, \mathrm{t}, J=8.3 \mathrm{~Hz}), 6.66(1 \mathrm{H}, \mathrm{dd}, J=8.3,2.4$ $\mathrm{Hz}), 6.70(1 / 2 \mathrm{H}, \mathrm{d}, J=2.4 \mathrm{~Hz}), 6.74(1 / 2 \mathrm{H}, \mathrm{d}, J=2.4 \mathrm{~Hz})$. HRMS $m / z$ : Calcd for $\mathrm{C}_{27} \mathrm{H}_{36} \mathrm{~N}_{2} \mathrm{O}_{11}: 564.2319$. Found: $564.2312 .[\alpha]_{\mathrm{D}}{ }^{24}+8.91^{\circ}(\mathrm{c}=0.10, \mathrm{MeOH})$.

1-( $\alpha$-D-Triacetylarabinopyranosyl)-2,3-dihydromelatonin (7) from $4-$ In the same procedure as described in the preparation of $\mathbf{5 a}, \mathrm{D}$-arabinose (208.2 mg, $1.39 \mathrm{mmol}), 4(64.9 \mathrm{mg}, 0.28 \mathrm{mmol}), \mathrm{MeOH}$ $(4.0 \mathrm{~mL})$, pyridine $(4.0 \mathrm{~mL}, 49.5 \mathrm{mmol})$, and $\mathrm{Ac}_{2} \mathrm{O}(2.0 \mathrm{~mL}, 21.2 \mathrm{mmol})$ were used. The acetylated mixture was subjected to PTLC on $\mathrm{SiO}_{2}$ developing twice with $\mathrm{CHCl}_{3}-\mathrm{MeOH}(97: 3, \mathrm{v} / \mathrm{v})$. Extraction of the band having an $R f$ value of $0.63-0.47$ with $\mathrm{CHCl}_{3}-\mathrm{MeOH}(95: 5, \mathrm{v} / \mathrm{v})$ gave 7 (129.6 mg, 95\%). 7: colorless oil. IR (film): 1747, $1655 \mathrm{~cm}^{-1} .{ }^{1} \mathrm{H}-\mathrm{NMR}\left(\mathrm{CDCl}_{3}\right) \delta: 1.60-1.80(2 \mathrm{H}, \mathrm{m}), 1.93$ (3/2H, s), 1.95 (3/2H, s), 1.99 (3/2H, s), 2.02 (3/2H, s), 2.03 (3/2H, s), 2.04 (3/2H, s), 2.17 (3/2H, s), 2.19 (3/2H, s), 3.17-3.48 (4H, m), 3.66-3.80 (2H, m), 3.74 (3H, s), 3.99 (1H, dd, J=13.0, $2.0 \mathrm{~Hz}), 4.76(1 / 2 \mathrm{H}, \mathrm{d}$, $J=9.0 \mathrm{~Hz}), 4.77(1 / 2 \mathrm{H}, \mathrm{d}, J=9.0 \mathrm{~Hz}), 5.13-5.18(1 \mathrm{H}, \mathrm{m}), 5.31(1 \mathrm{H}, \mathrm{m}), 5.41-5.45(1 \mathrm{H}, \mathrm{m}), 5.49(1 / 2 \mathrm{H}$, br $\mathrm{t}, J=5.2 \mathrm{~Hz}$, disappeared on addition of $\left.\mathrm{D}_{2} \mathrm{O}\right), 5.58(1 / 2 \mathrm{H}$, br $\mathrm{t}, J=5.2 \mathrm{~Hz}$, disappeared on addition of $\left.\mathrm{D}_{2} \mathrm{O}\right), 6.52(1 / 2 \mathrm{H}, \mathrm{d}, J=8.6 \mathrm{~Hz}), 6.53(1 / 2 \mathrm{H}, \mathrm{d}, J=8.6 \mathrm{~Hz}), 6.66(1 / 2 \mathrm{H}, \mathrm{dd}, J=8.6,2.7 \mathrm{~Hz}), 6.67(1 / 2 \mathrm{H}, \mathrm{dd}$, $J=8.6,2.7 \mathrm{~Hz}), 6.69(1 / 2 \mathrm{H}, \mathrm{d}, \quad J=2.7 \mathrm{~Hz}), 6.74(1 / 2 \mathrm{H}, \mathrm{d}, J=2.7 \mathrm{~Hz})$. HRMS $m / z$ : Calcd for $\mathrm{C}_{24} \mathrm{H}_{32} \mathrm{~N}_{2} \mathrm{O}_{9}$ : 492.2108. Found: $492.2098 .[\alpha]_{\mathrm{D}}{ }^{25}-38^{\circ}(\mathrm{c}=0.10, \mathrm{MeOH})$.

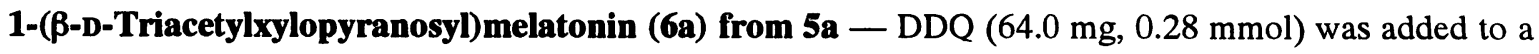
solution of $5 \mathbf{a}(115.7 \mathrm{mg}, 0.24 \mathrm{mmol})$ in dioxane $(5.0 \mathrm{~mL})$ and the mixture was stirred at $\mathrm{rt}$ for $0.5 \mathrm{~h}$. The 
whole was made alkaline by adding $8 \% \mathrm{NaHCO}_{3}$ under ice cooling and the whole was extracted with AcOEt. The extract was washed with brine, dried over $\mathrm{Na}_{2} \mathrm{SO}_{4}$ and evaporated under reduced pressure to leave an oil, which was subjected to PTLC on $\mathrm{SiO}_{2}$ developing three times with $\mathrm{CHCl}_{3}-\mathrm{MeOH}(98: 2$, $\mathrm{v} / \mathrm{v})$. Extraction of the band having an $R f$ value of $0.71-0.58$ with $\mathrm{CHCl}_{3}-\mathrm{MeOH}(95: 5, \mathrm{v} / \mathrm{v})$ gave $6 \mathbf{a}$ (65.0 mg, 56\%). 6a: pale yellow oil. IR (film): 1755, $1655 \mathrm{~cm}^{-1} .{ }^{1} \mathrm{H}-\mathrm{NMR}\left(\mathrm{CDCl}_{3}\right) \delta: 1.53(3 \mathrm{H}, \mathrm{s}), 1.93$ $(3 \mathrm{H}, \mathrm{s}), 2.05(3 \mathrm{H}, \mathrm{s}), 2.09(3 \mathrm{H}, \mathrm{s}), 2.77-2.84(1 \mathrm{H}, \mathrm{m}), 2.91-2.98(1 \mathrm{H}, \mathrm{m}), 3.39-3.46(1 \mathrm{H}, \mathrm{m}), 3.59$ $(1 \mathrm{H}, \mathrm{dd}, J=11.5,10.5 \mathrm{~Hz}), 3.66-3.74(1 \mathrm{H}, \mathrm{m}), 3.84(3 \mathrm{H}, \mathrm{s}), 4.29(1 \mathrm{H}, \mathrm{dd}, J=11.5,5.6 \mathrm{~Hz}), 5.19(1 \mathrm{H}$, ddd, $J=10.5,9.5,5.6 \mathrm{~Hz}), 5.29(1 \mathrm{H}, \mathrm{t}, J=9.0 \mathrm{~Hz}), 5.40(1 \mathrm{H}, \mathrm{d}, J=9.0 \mathrm{~Hz}), 5.44(1 \mathrm{H}, \mathrm{dd}, J=9.5,9.0 \mathrm{~Hz})$, $5.89\left(1 \mathrm{H}\right.$, br t, disappeared on addition of $\left.\mathrm{D}_{2} \mathrm{O}\right), 6.89(1 \mathrm{H}, \mathrm{dd}, J=8.8,2.4 \mathrm{~Hz}), 6.96(1 \mathrm{H}, \mathrm{d}, J=2.4 \mathrm{~Hz})$, $7.07(1 \mathrm{H}, \mathrm{s}), 7.23(1 \mathrm{H}, \mathrm{d}, J=8.8 \mathrm{~Hz})$. HRMS m/z: Calcd for $\mathrm{C}_{24} \mathrm{H}_{30} \mathrm{~N}_{2} \mathrm{O}_{9}: 490.1951$. Found: 490.1945 . $[\alpha]_{\mathrm{D}}{ }^{24}-37.0^{\circ}(\mathrm{c}=0.10, \mathrm{MeOH})$.

1-( $\beta$-D-Tetraacetylglucopyranosyl)melatonin (6b) from $5 b-$ In the same procedure as described in the preparation of $\mathbf{6 a}, \mathrm{DDQ}(84.2 \mathrm{mg}, 0.32 \mathrm{mmol}), \mathbf{5 b}(150.2 \mathrm{mg}, 0.27 \mathrm{mmol})$, and dioxane $(8.0 \mathrm{~mL})$ were used. The crude product was column-chromatographed on $\mathrm{SiO}_{2}$ with $\mathrm{CHCl}_{3}-\mathrm{MeOH}(99: 1 \mathrm{v} / \mathrm{v})$ to give $6 \mathbf{b}(130.7 \mathrm{mg}, 87 \%)$. 6b: yellow oil. IR (film): 1751, $1655 \mathrm{~cm}^{-1} .{ }^{1} \mathrm{H}-\mathrm{NMR}\left(\mathrm{CDCl}_{3}\right) \delta: 1.53(3 \mathrm{H}, \mathrm{s})$, $1.94(3 \mathrm{H}, \mathrm{s}), 2.03(3 \mathrm{H}, \mathrm{s}), 2.08(3 \mathrm{H}, \mathrm{s}), 2.09(3 \mathrm{H}, \mathrm{s}), 2.78-2.85(1 \mathrm{H}, \mathrm{m}), 2.92-2.98(1 \mathrm{H}, \mathrm{m})$, $3.40-3.47(1 \mathrm{H}, \mathrm{m}), 3.68-3.75(1 \mathrm{H}, \mathrm{m}), 3.85(3 \mathrm{H}, \mathrm{s}), 4.00(1 \mathrm{H}, \mathrm{ddd}, J=10.0,4.9,2.2 \mathrm{~Hz}), 4.17(1 \mathrm{H}$, dd, $J=12.5,2.2 \mathrm{~Hz}), 4.33(1 \mathrm{H}, \mathrm{dd}, J=12.5,4.9 \mathrm{~Hz}), 5.26(1 \mathrm{H}, \mathrm{dd}, J=10.0,9.5 \mathrm{~Hz}), 5.35(1 \mathrm{H}, \mathrm{dd}, J=9.5,9.0$ $\mathrm{Hz}), 5.45(1 \mathrm{H}, \mathrm{t}, J=9.5 \mathrm{~Hz}), 5.48(1 \mathrm{H}, \mathrm{d}, J=9.0 \mathrm{~Hz}), 5.92(1 \mathrm{H}, \mathrm{br} \mathrm{t}, J=5.0 \mathrm{~Hz}), 6.90(1 \mathrm{H}, \mathrm{dd}, J=8.9,2.3$ $\mathrm{Hz}), 6.97(1 \mathrm{H}, \mathrm{d}, J=2.3 \mathrm{~Hz}), 7.08(1 \mathrm{H}, \mathrm{s}), 7.23(1 \mathrm{H}, \mathrm{d}, J=8.9 \mathrm{~Hz})$. HRMS $m / z$ : Calcd for $\mathrm{C}_{27} \mathrm{H}_{34} \mathrm{~N}_{2} \mathrm{O}_{11}$ : 562.2163. Found: $562.2149 .[\alpha]_{\mathrm{D}}^{25}-29.7^{\circ}(\mathrm{c}=0.10, \mathrm{MeOH})$.

1-( $\beta$-D-Tetraacetylgalactopyranosyl)melatonin (6c) from $5 c-$ In the same procedure as described in the preparation of $6 \mathbf{a}, \mathrm{DDQ}(65.8 \mathrm{mg}, 0.29 \mathrm{mmol}), \mathbf{5 c}(136.2 \mathrm{mg}, 0.24 \mathrm{mmol})$, and dioxane $(8.0 \mathrm{~mL})$ were used. The crude product was column-chromatographed on $\mathrm{SiO}_{2}$ successively with $\mathrm{CHCl}_{3}-\mathrm{MeOH}$ (99:1 v/v) and AcOEt to give $6 \mathbf{c}(108.7 \mathrm{mg}, 80 \%)$. $6 \mathbf{c}$ : colorless oil. IR (film): 1751, $1655 \mathrm{~cm}^{-1} .{ }^{1} \mathrm{H}-\mathrm{NMR}$ $\left(\mathrm{CDCl}_{3}\right)$ 8: $1.55(3 \mathrm{H}, \mathrm{s}), 1.94(3 \mathrm{H}, \mathrm{s}), 2.01(3 \mathrm{H}, \mathrm{s}), 2.04(3 \mathrm{H}, \mathrm{s}), 2.26(3 \mathrm{H}, \mathrm{s}), 2.81-2.87(1 \mathrm{H}, \mathrm{m})$, $2.93-2.99(1 \mathrm{H}, \mathrm{m}), 3.40-3.47(1 \mathrm{H}, \mathrm{m}), 3.68-3.75(1 \mathrm{H}, \mathrm{m}), 3.85(3 \mathrm{H}, \mathrm{s}), 4.17(1 \mathrm{H}, \mathrm{dd}, J=13.5,9.0$ $\mathrm{Hz}), 4.20$ (1H, dd, $J=9.0,5.0 \mathrm{~Hz}), 4.22(1 \mathrm{H}, \mathrm{dd}, J=13.5,5.0 \mathrm{~Hz}), 5.27(1 \mathrm{H}, \mathrm{dd}, J=10.4,3.2 \mathrm{~Hz}), 5.44(1 \mathrm{H}$, d, $J=9.0 \mathrm{~Hz}), 5.51(1 \mathrm{H}, \mathrm{dd}, J=10.4,9.0 \mathrm{~Hz}), 5.56(1 \mathrm{H}, \mathrm{d}, J=3.2 \mathrm{~Hz}), 5.92(1 \mathrm{H}, \mathrm{br} \mathrm{t}, J=5.0 \mathrm{~Hz}), 6.90(1 \mathrm{H}$, $\mathrm{dd}, J=8.8,2.5 \mathrm{~Hz}), 6.97(1 \mathrm{H}, \mathrm{d}, J=2.5 \mathrm{~Hz}), 7.12(1 \mathrm{H}, \mathrm{s}), 7.27(1 \mathrm{H}, \mathrm{d}, J=8.8 \mathrm{~Hz}) . \mathrm{HRMS} m / z$ : Calcd for $\mathrm{C}_{27} \mathrm{H}_{34} \mathrm{~N}_{2} \mathrm{O}_{11}: 562.2163$. Found: 562.2163. $[\alpha]_{\mathrm{D}}{ }^{25}-22^{\circ}(\mathrm{c}=0.10, \mathrm{MeOH})$.

1-( $\alpha$-D-Triacetylarabinopyranosyl)melatonin (3a) and 3-[2-(Acetylamino)acetyl]-1-( $\alpha$-D-triacetylarabinopyranosyl)-5-methoxyindole (8a) from 7 - [Entry 1] In the same procedure as described in 
the preparation of $6 \mathrm{a}, \mathrm{DDQ}(65.8 \mathrm{mg}, 0.29 \mathrm{mmol}), 7(136.2 \mathrm{mg}, 0.24 \mathrm{mmol})$, and dioxane $(8.0 \mathrm{~mL})$ were used and stirring was continued for $1 \mathrm{~h}$. The crude product was subjected to PTLC on $\mathrm{SiO}_{2}$ developing twice with $\mathrm{CHCl}_{3}-\mathrm{MeOH}-28 \% \mathrm{NH}_{3}(46: 1: 0.1, \mathrm{v} / \mathrm{v})$. Extraction of the band having an $R f$ value of $0.63-0.52$ with $\mathrm{CHCl}_{3}-\mathrm{MeOH}(95: 5, \mathrm{v} / \mathrm{v})$ gave $3 \mathbf{a}(62.1 \mathrm{mg}, 43 \%)$. Extraction of the band having an $R f$ value of $0.37-0.32$ with $\mathrm{CHCl}_{3}-\mathrm{MeOH}(95: 5$, v/v) gave $8 \mathbf{a}(15.0 \mathrm{mg}, 10 \%)$. 3a: colorless oil. IR (film): 1749, $1655 \mathrm{~cm}^{-1} .{ }^{1} \mathrm{H}-\mathrm{NMR}\left(\mathrm{CDCl}_{3}\right)$ ס: $1.55(3 \mathrm{H}, \mathrm{s}), 1.94(3 \mathrm{H}, \mathrm{s}), 2.04(3 \mathrm{H}, \mathrm{s}), 2.26(3 \mathrm{H}, \mathrm{s}), 2.80-2.87$ $(1 \mathrm{H}, \mathrm{m}), 2.93-2.99(1 \mathrm{H}, \mathrm{m}), 3.39-3.47(1 \mathrm{H}, \mathrm{m}), 3.68-3.76(1 \mathrm{H}, \mathrm{m}), 3.85(3 \mathrm{H}, \mathrm{s}), 3.93(1 \mathrm{H}$, dd, $J=13.4,0.7 \mathrm{~Hz}), 4.20(1 \mathrm{H}$, dd, $J=13.4,2.0 \mathrm{~Hz}), 5.27(1 \mathrm{H}, \mathrm{dd}, J=10.3,3.4 \mathrm{~Hz}), 5.35(1 \mathrm{H}, \mathrm{d}, J=9.0 \mathrm{~Hz})$, $5.44(1 \mathrm{H}, \mathrm{ddd}, J=3.4,2.0,0.7 \mathrm{~Hz}), 5.55(1 \mathrm{H}, \mathrm{dd}, J=10.3,9.0 \mathrm{~Hz}), 5.92(1 \mathrm{H}, \mathrm{br} \mathrm{t}, J=5.5 \mathrm{~Hz}$ disappeared on addition of $\left.\mathrm{D}_{2} \mathrm{O}\right), 6.89(1 \mathrm{H}, \mathrm{dd}, J=9.0,2.4 \mathrm{~Hz}), 6.97(1 \mathrm{H}, \mathrm{d}, J=2.4 \mathrm{~Hz}), 7.13(1 \mathrm{H}, \mathrm{s}), 7.26(1 \mathrm{H}, \mathrm{d}, J=9.0$ $\mathrm{Hz}$ ). HRMS $m / z$ : Calcd for $\mathrm{C}_{24} \mathrm{H}_{30} \mathrm{~N}_{2} \mathrm{O}_{9}: 490.1951$. Found: 490.1952 . $[\alpha]_{\mathrm{D}}{ }^{26}+2.78^{\circ}(\mathrm{c}=0.09, \mathrm{MeOH})$.

[Entry 2] In the same procedure as described in the preparation of $6 \mathrm{a}, \mathrm{DDQ}(66.8 \mathrm{mg}, 0.29 \mathrm{mmol}), 7$ $(72.4 \mathrm{mg}, 0.15 \mathrm{mmol})$, and dioxane $(5.0 \mathrm{~mL})$ were used. After the same work-up and separation as described in the Entry 1, 3a (3.1 mg, 4\%) and $8 \mathbf{a}(25.5 \mathrm{mg}, 34 \%)$ were obtained. 8a: colorless oil. IR (film): 1749, $1653 \mathrm{~cm}^{-1} .{ }^{1} \mathrm{H}-\mathrm{NMR}\left(\mathrm{CDCl}_{3}\right)$ 8: $1.66(3 \mathrm{H}, \mathrm{s}), 2.04(3 \mathrm{H}, \mathrm{s}), 2.11(3 \mathrm{H}, \mathrm{s}), 2.33(3 \mathrm{H}, \mathrm{s}), 3.89$ $(3 \mathrm{H}, \mathrm{s}), 3.96(1 \mathrm{H}, \mathrm{dd}, J=13.4,1.0 \mathrm{~Hz}), 4.25(1 \mathrm{H}, \mathrm{dd}, J=13.4,2.0 \mathrm{~Hz}), 4.63(1 \mathrm{H}, \mathrm{dd}, J=18.0,4.0 \mathrm{~Hz}), 4.68$ $(1 \mathrm{H}, \mathrm{dd}, J=18.0,4.5 \mathrm{~Hz}), 5.29$ (1H, dd, $J=10.0,3.4 \mathrm{~Hz}), 5.43(1 \mathrm{H}, \mathrm{d}, J=9.0 \mathrm{~Hz}), 5.48(1 \mathrm{H}, \mathrm{ddd}, J=3.4,2.0$, $1.0 \mathrm{~Hz}), 5.62(1 \mathrm{H}, \mathrm{dd}, J=10.0,9.0 \mathrm{~Hz}), 6.66\left(1 \mathrm{H}\right.$, br t, disappeared on addition of $\left.\mathrm{D}_{2} \mathrm{O}\right), 6.98(1 \mathrm{H}$, dd, $J=9.0,2.4 \mathrm{~Hz}), 7.38(1 \mathrm{H}, \mathrm{d}, J=9.0 \mathrm{~Hz}), 7.82(1 \mathrm{H}, \mathrm{d}, J=2.4 \mathrm{~Hz}), 8.03(1 \mathrm{H}, \mathrm{s})$. HRMS (FAB $\left.{ }^{+}\right) \mathrm{m} / z$ : Calcd for $\mathrm{C}_{24} \mathrm{H}_{29} \mathrm{~N}_{2} \mathrm{O}_{10}: 505.1823\left(\mathrm{MH}^{+}\right)$. Found: $505.1821 .[\alpha]_{\mathrm{D}}^{28}+3.88^{\circ}(\mathrm{c}=0.12, \mathrm{MeOH})$.

1-( $\beta$-D-Xylopyranosyl)melatonin (2a) from $6 \mathbf{a}-8 \% \mathrm{NaOH}(0.5 \mathrm{~mL})$ was added to a solution of $\mathbf{6 a}$ $(29.9 \mathrm{mg}, 0.06 \mathrm{mmol})$ in $\mathrm{MeOH}(1.0 \mathrm{~mL})$ and the mixture was stirred at $\mathrm{rt}$ for $1 \mathrm{~h}$. After evaporation of the solvent under reduced pressure, brine was added to the residue and the whole was extracted with AcOEt-MeOH $(95: 5, \mathrm{v} / \mathrm{v})$. The extract was dried over $\mathrm{Na}_{2} \mathrm{SO}_{4}$ and evaporated under reduced pressure to leave an oil, which was column-chromatographed on $\mathrm{SiO}_{2}$ with $\mathrm{AcOEt}-\mathrm{MeOH}(97: 3$, v/v) to give $2 \mathbf{a}$ (18.5 mg, 83\%). 2a: colorless oil. IR (film): 3321, 2920, 1633, 1483, 1454, 1232, $1051 \mathrm{~cm}^{-1}$. ${ }^{1} \mathrm{H}-\mathrm{NMR}$ (DMSO-d $)_{6} \delta: 1.81(3 \mathrm{H}, \mathrm{s}), 2.75(2 \mathrm{H}, \mathrm{t}, J=7.6 \mathrm{~Hz}), 3.33(2 \mathrm{H}, \mathrm{t}, J=7.6 \mathrm{~Hz}), 3.34-3.41(2 \mathrm{H}, \mathrm{m})$, $3.44-3.51(1 \mathrm{H}, \mathrm{m}), 3.68(1 \mathrm{H}, \mathrm{dt}, J=5.6,8.8 \mathrm{~Hz}), 3.74-3.79(1 \mathrm{H}, \mathrm{m}), 3.77(3 \mathrm{H}, \mathrm{s}), 5.08(1 \mathrm{H}, \mathrm{d}, J=4.6$ $\mathrm{Hz}$, disappeared on addition of $\left.\mathrm{D}_{2} \mathrm{O}\right), 5.10\left(1 \mathrm{H}, \mathrm{d}, J=5.6 \mathrm{~Hz}\right.$, disappeared on addition of $\left.\mathrm{D}_{2} \mathrm{O}\right), 5.14(1 \mathrm{H}$, $\mathrm{d}, J=4.6 \mathrm{~Hz}$, disappeared on addition of $\left.\mathrm{D}_{2} \mathrm{O}\right), 5.21(1 \mathrm{H}, \mathrm{d}, J=9.0 \mathrm{~Hz}), 6.76(1 \mathrm{H}, \mathrm{dd}, J=8.8,2.4 \mathrm{~Hz}), 7.02$ $(1 \mathrm{H}, \mathrm{d}, J=2.4 \mathrm{~Hz}), 7.20(1 \mathrm{H}, \mathrm{s}), 7.37(1 \mathrm{H}, \mathrm{d}, J=8.8 \mathrm{~Hz}), 7.91\left(1 \mathrm{H}\right.$, br t, disappeared on addition of $\left.\mathrm{D}_{2} \mathrm{O}\right)$. HRMS $m / z$ : Calcd for $\mathrm{C}_{18} \mathrm{H}_{24} \mathrm{~N}_{2} \mathrm{O}_{6}$ : 364.1634. Found: 364.1629. $[\alpha]_{\mathrm{D}}{ }^{25}-22.0^{\circ}(\mathrm{c}=0.093, \mathrm{MeOH})$. 
1-( $\alpha$-D-Arabinopyranosyl)melatonin (3b) from 3a - In the same procedure as described in the preparation of $2 \mathbf{a}, 8 \% \mathrm{NaOH}(0.5 \mathrm{~mL}), 3 \mathbf{a}(28.7 \mathrm{mg}, 0.06 \mathrm{mmol})$, and $\mathrm{MeOH}(1.0 \mathrm{~mL})$ were used and stirring was continued for $15 \mathrm{~min}$. The crude product was subjected to PTLC on $\mathrm{SiO}_{2}$ developing twice with $\mathrm{CHCl}_{3}-\mathrm{MeOH}(85: 15, \mathrm{v} / \mathrm{v})$. Extraction of the band having an $R f$ value of $0.59-0.49$ with $\mathrm{CHCl}_{3}-\mathrm{MeOH}(85: 15$, v/v) gave $3 \mathbf{b}$ (18.5 mg, 87\%). 3b: colorless oil. IR (film): 3354, 2933, 1641, 1558, 1485, 1232, $1085 \mathrm{~cm}^{-1} .{ }^{1} \mathrm{H}-\mathrm{NMR}\left(\mathrm{CD}_{3} \mathrm{OD}\right) \delta: 1.91(3 \mathrm{H}, \mathrm{s}), 2.88(2 \mathrm{H}, \mathrm{t}, J=7.1 \mathrm{~Hz}), 3.46(2 \mathrm{H}$, br t,$J=7.1$ Hz), 3.70 (1H, dd, $J=9.4,3.3 \mathrm{~Hz}), 3.83(1 \mathrm{H}, \mathrm{dd}, J=12.9,1.2 \mathrm{~Hz}), 3.83(3 \mathrm{H}, \mathrm{s}), 3.97(1 \mathrm{H}, \mathrm{ddd}, J=3.3,2.4$, $1.2 \mathrm{~Hz}), 3.98(1 \mathrm{H}, \mathrm{dd}, J=12.9,2.4 \mathrm{~Hz}), 4.22(1 \mathrm{H}, \mathrm{t}, J=9.0 \mathrm{~Hz}), 5.18(1 \mathrm{H}, \mathrm{d}, J=9.0 \mathrm{~Hz}), 6.80(1 \mathrm{H}, \mathrm{dd}$, $J=9.0,2.4 \mathrm{~Hz}), 7.05(1 \mathrm{H}, \mathrm{d}, J=2.4 \mathrm{~Hz}), 7.23(1 \mathrm{H}, \mathrm{s}), 7.44(1 \mathrm{H}, \mathrm{d}, J=9.0 \mathrm{~Hz})$. HRMS (FAB $\left.{ }^{+}\right) m / z$ : Calcd for $\mathrm{C}_{18} \mathrm{H}_{25} \mathrm{~N}_{2} \mathrm{O}_{6}: 365.1713\left(\mathrm{MH}^{+}\right)$. Found: 365.1727 . $[\alpha]_{\mathrm{D}}{ }^{26}-15.5^{\circ}(\mathrm{c}=0.10, \mathrm{MeOH})$.

1-( $\beta$-D-Glucopyranosyl)melatonin (2b) from $\mathbf{6 b}-$ In the same procedure as described in the preparation of $2 \mathbf{a}, 8 \% \mathrm{NaOH}(0.5 \mathrm{~mL}), 6 \mathbf{b}(18.5 \mathrm{mg}, 0.03 \mathrm{mmol})$, and $\mathrm{MeOH}(1.0 \mathrm{~mL})$ were used and stirring was continued for $0.5 \mathrm{~h}$. The crude product was column-chromatographed with AcOEt-MeOH (95:5 v/v) to give $\mathbf{2 b}(11.7 \mathrm{mg}, 90 \%)$. 2b: yellow oil. IR (film): 3392, 2924, 1635, 1485, 1074, $1032 \mathrm{~cm}^{-1}$. ${ }^{1} \mathrm{H}-\mathrm{NMR}\left(\mathrm{CD}_{3} \mathrm{OD}\right) \delta: 1.91(3 \mathrm{H}, \mathrm{s}), 2.88(2 \mathrm{H}, \mathrm{t}, J=7.3 \mathrm{~Hz}), 3.45(2 \mathrm{H}, \mathrm{t}, J=7.3 \mathrm{~Hz}), 3.47(1 \mathrm{H}, \mathrm{dd}, J=9.5$, $8.8 \mathrm{~Hz}), 3.54(1 \mathrm{H}$, ddd, $J=9.5,5.0,2.0 \mathrm{~Hz}), 3.57(1 \mathrm{H}, \mathrm{dd}, J=9.0,8.8 \mathrm{~Hz}), 3.69(1 \mathrm{H}, \mathrm{dd}, J=12.1,5.6 \mathrm{~Hz})$, $3.82(3 \mathrm{H}, \mathrm{s}), 3.86(1 \mathrm{H}, \mathrm{dd}, J=12.2,2.0 \mathrm{~Hz}), 3.88(1 \mathrm{H}, \mathrm{t}, J=9.0 \mathrm{~Hz}), 4.52(1 \mathrm{H}$, br s), $5.34(1 \mathrm{H}, \mathrm{d}, J=9.0$ $\mathrm{Hz}), 6.81(1 \mathrm{H}, \mathrm{dd}, J=8.8,2.4 \mathrm{~Hz}), 7.05(1 \mathrm{H}, \mathrm{d}, J=2.4 \mathrm{~Hz}), 7.21(1 \mathrm{H}, \mathrm{s}), 7.39$ (1H, d, $J=8.8 \mathrm{~Hz})$. HRMS $\left(\mathrm{FAB}^{+}\right) \mathrm{m} / z$ : Calcd for $\mathrm{C}_{19} \mathrm{H}_{27} \mathrm{~N}_{2} \mathrm{O}_{7}: 365.1819\left(\mathrm{MH}^{+}\right)$. Found: $365.1823 .[\alpha]_{\mathrm{D}}{ }^{27}-16.8^{\circ}(\mathrm{c}=0.10, \mathrm{MeOH})$. 1-( $\beta$-D-Galactopyranosyl)melatonin (2c) from $\boldsymbol{6} c$ - In the same procedure as described in the preparation of $2 \mathbf{a}, 8 \% \mathrm{NaOH}(0.5 \mathrm{~mL}), \mathbf{6} \mathbf{c}(44.5 \mathrm{mg}, 0.08 \mathrm{mmol})$, and $\mathrm{MeOH}(1.0 \mathrm{~mL})$ were used and stirring was continued for $15 \mathrm{~min}$. Column-chromatography was performed with $\mathrm{CHCl}_{3}-\mathrm{MeOH}(85: 15$ v/v) to give 2c (29.1 mg, 93\%). 2c: colorless oil. IR (film): 3363, 2927, 1633, 1483, 1088, $1041 \mathrm{~cm}^{-1}$. ${ }^{1} \mathrm{H}-$ NMR (CD $\mathrm{OD}) \delta: 1.91(3 \mathrm{H}, \mathrm{s}), 2.88(2 \mathrm{H}, \mathrm{t}, J=7.1 \mathrm{~Hz}), 3.46(2 \mathrm{H}$, br t,$J=7.1 \mathrm{~Hz}), 3.68(1 \mathrm{H}, \mathrm{dd}, J=9.0,2.4$ $\mathrm{Hz}), 3.71-3.79(3 \mathrm{H}, \mathrm{m}), 3.82(3 \mathrm{H}, \mathrm{s}), 3.99(1 \mathrm{H}, \mathrm{d}, J=2.4 \mathrm{~Hz}), 4.22(1 \mathrm{H}, \mathrm{t}, J=9.0 \mathrm{~Hz}), 5.27(1 \mathrm{H}, \mathrm{d}, J=9.0$ $\mathrm{Hz}), 6.80(1 \mathrm{H}, \mathrm{dd}, J=8.8,2.4 \mathrm{~Hz}), 7.05(1 \mathrm{H}, \mathrm{d}, J=2.4 \mathrm{~Hz}), 7.25(1 \mathrm{H}, \mathrm{s}), 7.46(1 \mathrm{H}, \mathrm{d}, J=8.8 \mathrm{~Hz})$. HRMS $\left(\mathrm{FAB}^{+}\right) \mathrm{m} / z$ : Calcd for $\mathrm{C}_{19} \mathrm{H}_{27} \mathrm{~N}_{2} \mathrm{O}_{7}: 395.1818\left(\mathrm{MH}^{+}\right)$. Found: $395.1807 .[\alpha]_{\mathrm{D}}{ }^{27}-8.6^{\circ}(\mathrm{c}=0.10, \mathrm{MeOH})$.

3-[2-(Acetylamino)acetyl]-1-( $\alpha$-D-arabinopyranosyl)-5-methoxyindole (8b) from 8a - In the same procedure as described in the preparation of $\mathbf{2 a}, 8 \% \mathrm{NaOH}(0.5 \mathrm{~mL}), \mathbf{8 a}(10.6 \mathrm{mg}, 0.02 \mathrm{mmol})$, and $\mathrm{MeOH}(1.0 \mathrm{~mL})$ were used and stirring was continued for $30 \mathrm{~min}$. Column-chromatography was performed with $\mathrm{CHCl}_{3}-\mathrm{MeOH}(85: 15 \mathrm{v} / \mathrm{v})$ to give $\mathbf{8 b}(6.4 \mathrm{mg}, 81 \%)$. 8b: colorless oil. IR (film): 3398 , 2924, 1641, 1525, $1086 \mathrm{~cm}^{-1} .{ }^{1} \mathrm{H}-\mathrm{NMR}\left(\mathrm{CD}_{3} \mathrm{OD}\right) \delta: 2.07(3 \mathrm{H}, \mathrm{s}), 3.72(1 \mathrm{H}, \mathrm{dd}, J=9.0,3.3 \mathrm{~Hz}), 3.84(3 \mathrm{H}$, s), $3.87(1 \mathrm{H}, \mathrm{dd}, J=12.4,1.1 \mathrm{~Hz}), 3.99(1 \mathrm{H}$, ddd, $J=3.3,2.0,1.1 \mathrm{~Hz}), 4.04(1 \mathrm{H}, \mathrm{dd}, J=12.4,2.0 \mathrm{~Hz}), 4.22$ 
$(1 \mathrm{H}, \mathrm{t}, J=9.0 \mathrm{~Hz}), 4.54(1 \mathrm{H}, \mathrm{d}, J=17.5 \mathrm{~Hz}), 4.61(1 \mathrm{H}, \mathrm{d}, J=17.5 \mathrm{~Hz}), 5.30(1 \mathrm{H}, \mathrm{d}, J=9.0 \mathrm{~Hz}), 6.90(1 \mathrm{H}, \mathrm{dd}$, $J=9.0,2.4 \mathrm{~Hz}), 7.62(1 \mathrm{H}, \mathrm{d}, J=9.0 \mathrm{~Hz}), 7.77(1 \mathrm{H}, \mathrm{d}, J=2.4 \mathrm{~Hz}), 8.39(1 \mathrm{H}, \mathrm{s})$. HRMS (FAB $\left.{ }^{+}\right) m / z$ : Calcd for $\mathrm{C}_{18} \mathrm{H}_{23} \mathrm{~N}_{2} \mathrm{O}_{7}: 379.1506\left(\mathrm{MH}^{+}\right)$. Found: 379.1499 .

\section{REFERENCES AND NOTES}

1. a) This is Part 118 of a series entitled "The Chemistry of Indoles". b) Part 117: M. Somei and M. Seto, Heterocycles, 2003, 60, 519. c) M. Somei, F. Yamada, J. Kato, Y. Suzuki, and Y. Ueda, ibid., $2002,56,81$.

2. A. B. Lerner, J. D. Case, Y. Takahashi, T. H. Lee, and W. Mori, J. Am. Chem. Soc., 1958, 80, 2587; J. Arendt, M. Aldhouse, and V. Marks, Br. Med.J., 1986, 292, 1170; S. M. Reppert, D. R. Weaver, S. A. Rivkees, and E. G. Stopa, Science, 1988, 242, 78.

3. a) M. Pappolla, P. Bozner, C. Soto, H. Shao, N. K. Robakis, M. Zagorski, B. Frangione, and J. Ghiso, J. Biol. Chem., 1998, 273, 7185; b) M. Loeffler, Exp. Clin. Endocrinol. Diabetes, 1996, 104, 308; c) S. -W. Ying, L. P. Niles, and C. Crocker, Eur. J. Pharmacol., Mol. Pharmacol. Sect., 1993, 246, 89; d) H. Schmid, Gerontology, 1993, 39, 189; e) F. Waldhauser, B. Ehrart, and E. Forster, Experientia, 1993, 49, 671; f) W. Pierpaoli, Aging, 1991, 3, 99.

4. a) M. Somei and T. Kawasaki, Heterocycles, 1989, 29, 1251; b) M. Somei and Y. Fukui, ibid., 1993, 36, 1859; c) M. Somei, F. Yamada, and H. Morikawa, ibid., 1997, 46, 91; d) M. Somei, N. Oshikiri, M. Hasegawa, and F. Yamada, ibid., 1999, 51, 1237; e) M. Somei, Y. Fukui, M. Hasegawa, N. Oshikiri, and T. Hayashi, ibid., 2000, 53, 1725.

5. M. N. Preobrazhenskaya, Usp. Khim., 1967, 36, 1760 (Chem. Abstr., 1968, 68, 104846); M. N. Preobrazhenskaya and I. A. Korbukh, "Chemistry of Nucleosides and Nucleotides", Vol. 3, ed. by L. B. Townsend, Plenum Press, New York, 1994, pp.1—99 and references cited therein.

6. M. Somei, K. Yamada, M. Hasegawa, M. Tabata, Y. Nagahama, H. Morikawa, and F. Yamada, Heterocycles, 1996, 43, 1855.

7. A. E. Lanzilotti, R. Littell, W. J. Fanshawe, T. C. McKenzie, and F. M. Lovell, J. Org. Chem., 1979, 44, 4809 . 